\title{
The Effect of Multilevel Surgery on the Gait Pattern of Children with Hereditary Spastic Paraplegia, are there Differences in Outcome Compared to Those with Bilateral Spastic Cerebral Palsy?
}

Sandra Prinsen, $M D^{1^{*}}$, Charlotte Buyck, $P T^{2}$, Nathalie de Beukelaer, $P T^{3,4}$, Lynn Bar On, PhD ${ }^{3,5}$, Els Ortibus, MD, PhD ${ }^{6}$, Kaat Desloovere, $M D$, PhD ${ }^{3,4}$, and Anja Van Campenhout, MD, PhD ${ }^{1}$

${ }^{1}$ Department of Orthopaedics, University Hospital Leuven, Leuven, Belgium

${ }^{2}$ Department of Rehabilitation Sciences, Research Group for Neurorehabilitation, Leuven, Belgium

${ }^{3}$ Department of Rehabilitation Sciences, KU Leuven, Belgium

${ }^{4}$ Clinical Motion Analysis Laboratory, University Hospital Leuven, Leuven, Belgium

${ }^{5}$ Department of Rehabilitation Medicine, Amsterdam University Medical Center, Location VUmc, Amsterdam, Netherlands ${ }^{6}$ Department of Development and Regeneration, KU Leuven, Organ Systems, Leuven, Belgium

\begin{abstract}
Background: Hereditary spastic paraplegia (HSP) is a group of neurodegenerative genetic disorders with clinical similarity to spastic diplegic Cerebral Palsy (CP). Multilevel surgery (MLS) is frequently used to treat the secondary musculoskeletal problems in patients with neuromuscular disorders.
\end{abstract}

Research question: The aim of this retrospective study was to assess the short- and medium-term effects of MLS using 3D gait analysis in children with HSP, if there are any parameters that can predict a good outcome and further a comparison with a group of children with spastic diplegic CP to assess if children with HSP should be treated differently.

Methods: The 3D gait kinematics, including calculation of the movement analysis profile (MAPS) per joint and the overall gait profile score (GPS), of 15 children with HSP were compared pre and with a mean of $11.6 \pm 2$ months (short-term follow-up) post-MLS. In 9 of the children, gait was additionally analysed $33.7 \pm 9$ months (medium term follow-up) postMLS. These medium-term follow-up results were compared to the post-operative results of a group of 9 children with spastic diplegic CP who had also undergone MLS at a similar age.

Results: GPS, GPS in the sagittal and in the transverse plane, minimal and maximal hip rotation and angle of the hip and knee at initial contact improved at medium-term FU. There was no change in pelvic tilt and in maximum hip extension during stance. Largest decrease in GPS at medium-term was predicted by high pre-operative values of GPS and GPS in the sagittal plane, large angles of hip and knee flexion during stance phase and at initial contact. Only mean pelvic rotation was significantly higher post-MLS in children with CP.

Conclusion: MLS in children with HSP improved gait in short- and medium-term, and were comparable with the effects of MLS in CP.

\section{Background}

Hereditary spastic paraplegia (HSP) is a group of genetic disorders in which the neurons of the spinal cord are affected. In Europe, the prevalence is estimated to be 3 to 4 per 100000 live births [1]. The main symptoms are spasticity and weakness of the lower limbs [1-3]. There is a very wide range of ages of onset, rate of progression and degree of disability. A differential diagnosis for HSP with early onset is cerebral palsy (CP), which is defined as an 'umbrella term covering a group of non-progressive, but often changing motor impair-
*Corresponding author: Sandra Prinsen, MD, Department of Orthopaedics, University Hospital Leuven, Leuven, Belgium

Accepted: October 20, 2020

Published online: October 22, 2020

Citation: Prinsen S, Buyck C, Beukelaer N, et al. (2020) The Effect of Multilevel Surgery on the Gait Pattern of Children with Hereditary Spastic Paraplegia, are there Differences in Outcome Compared to Those with Bilateral Spastic Cerebral Palsy?. J Orthop Surg Tech 3(2):179-190 
Citation: Prinsen S, Buyck C, Beukelaer N, et al. (2020) The Effect of Multilevel Surgery on the Gait Pattern of Children with Hereditary Spastic Paraplegia, are there Differences in Outcome Compared to Those with Bilateral Spastic Cerebral Palsy?. J Orthop Surg Tech 3(2):179-190

ment syndromes secondary to lesions or anomalies of the brain arising in the early stages of its development' [4]. Prematurity and low birth weight are the highest risk factors for $\mathrm{CP}$, although, recently, there is more and more evidence for genetic mechanisms also playing a role in the etiology of $C P$ [5]. HSP is typically diagnosed when symptoms of spasticity and weakness in the lower limbs are present, when there is possible evidence of a family history and when risk factors leading to a clinical picture of CP are absent. Genetic testing is mandatory to confirm the hypothesis of HSP and more than $50 \%$ of the dominant inherited HSP are caused by mutations on the spastin 4 and spataxin gene. However, there is a wide range in genetic causes and besides $\mathrm{X}$-linked of recessive forms exist [6]. In uncomplicated or pure forms of HSP, spasticity and weakness are limited to the lower extremities but sensory disturbances or urinary dysfunction can be present. In its complicated form, HSP presents with additional symptoms such as ataxia, dementia, seizures, cognitive impairment and peripheral neuropathy [6].

Although the clinical pictures of HSP and CP may resemble each other in part, there are two major differences: 1 ) CP presents with a brain lesion explaining the clinical findings, whereas this is not the case for HSP and 2) HSP can be progressive while $\mathrm{CP}$ is a non-progressive neurological disorder. The spasticity, muscle weakness and sensory deficits lead to muscle shortening, gait deterioration and eventually to bony torsions and deformities, known as secondary impairments [7] While many symptomatic treatment modalities exist and are known to be effective for children with $C P$, little is known about the effect of the same treatments in HSP [8]. Until now, there is no curative method for either HSP or CP and only symptomatic treatments are available. It has been demonstrated that intrathecal baclofen pumps and injections with botulinum toxin Type a decrease muscle spasticity and that multilevel surgery (MLS) can be used to treat the secondary deformities. MLS is defined as surgery that involves at least two or more soft-tissue or bony surgical procedures at two or more anatomical levels during one operative procedure, requiring only one hospital admission and one period of rehabilitation [9]. Good long-term effects of MLS on both gait and function in children with $C P$ have been reported. Since gait disturbance is one of the first symptoms observed in HSP and $C P$, a 3 dimensional gait analysis ( 3 DGA) can be used to quantify gait deviations with spatiotemporal parameters, joint kinematics and kinetics, and electromyography [10]. A significant increase in maximum knee flexion, ankle dorsiflexion and in walking distance was found [11-13].

Although the clinical symptoms of non-progressive HSP and bilateral spastic $\mathrm{CP}$ are often similar and lead to the same secondary orthopedic lower limb and gait problems, so far, to the author's knowledge, there are no studies available on the effect of MLS on the gait of patients with HSP.

Therefore, the research questions of the current study were: (1) What are the effects of MLS on the gait pattern of children with HSP in short-and middle term follow-up? (2) Are there differences in outcome post-MLS in HSP children compared to children with bilateral spastic CP? (3) Is it possible to explore potential predictors for a good outcome after MLS in children with HSP.

\section{Methods}

A retrospective cohort study was performed at the University Hospitals of Leuven (UH Leuven). The database of clinical motion analysis laboratory (CMAL) at the University Hospitals of Leuven was used to retrospectively identify subjects to be included in this study when they: 1) Had a diagnosis of HSP, 2) Had had MLS at UH Leuven and 3) Had 3DGA pre- and at least once post-MLS. All available postoperative gait analyses were included provided no additional MLS took place after the initial MLS and the subject had not undergone a selective dorsal rhizotomy (SDR) during follow-up (FU). For this study, MLS was defined as one episode of surgery with at least two or more bony or soft-tissue procedures (botulinum toxin-A injections included) at two or more anatomical levels (hip, knee or ankle), followed by one period of rehabilitation [9]. Using static and dynamic evaluation, one of two experienced orthopaedic surgeons decided which procedures needed to be performed on every individual patient. Postoperative rehabilitation was guided by a team of physiotherapists at the UH Leuven. For those subjects with HSP who had a GA at medium-term follow-up, (more than 1.5 years post-operatively), a matched patient with bilateral spastic $C P$, who also had undergone MLS, was retrospectively selected from the same CMAL database. Matching was performed for age, Gross Motor Function Classification system (GMFCS) level and type of MLS.

Evaluation of mobility using goniometry to define muscle contractures and bony deformities, spasticity by means modified Ashworth [14] and modified Tardieu [15] scales and muscle strength (manual muscle testing) and selectivity was performed. Those, and GAs were carried out by trained clinicians as part of standardized hospital care.

During GA, subjects walked 10 meters along a walkway at a self-selected walking pace. The data were collected using a 16 camera VICON system, operating at $1000 \mathrm{~Hz}$, with 15 reflective markers located at specific anatomical landmarks of the lower limbs, according to the lower limb Vicon Plugin Gait marker configuration (VICON, Oxford Metrics, Oxford, UK).

GAs were divided into short- and medium-term FU visits. A first GA was performed about one year after surgery. For the patients with a longer follow-up, the most recent GA was selected. Gait analyses were processed with Vicon Nexus (version 1.8.5 for data that are collected after January 2007, version 1.5.2 for the data collected between November 2005 and December 2006 and using Vicon Workstation version 5.1, for data collected before November 2005).

3D kinematic data were derived bilaterally for the pelvis, hip, knee, ankle and foot in the sagittal, coronal and transverse plane, bilaterally. Gait cycles were determined by indicating the events of initial contact and toe off using Nexus software (Vicon, Oxford Metrics Group, Oxford, UK). This was done for all the trials. An additional visual quality control, with a check for lack of marker visibility and/or obvious artefacts, of all kinematic data was performed in custom-made MATLAB software (Mathworks 1, Natick, MA, USA), prior to further data analysis. 
Citation: Prinsen S, Buyck C, Beukelaer N, et al. (2020) The Effect of Multilevel Surgery on the Gait Pattern of Children with Hereditary Spastic Paraplegia, are there Differences in Outcome Compared to Those with Bilateral Spastic Cerebral Palsy?. J Orthop Surg Tech 3(2):179-190

Primary outcome measure was the gait profile score (GPS), a summary statistic for the degree of gait deviation [16]. The GPS is the root mean square (RMS) difference between a set of Motion Analysis Profile (MAPs) from the child and from a database of typically developing (TD) children. The MAPs is the average RMS difference over the gait cycle at two percent intervals across the gait cycle, i.e. 51 points, between the child's kinematic curve and the average of the TD children database. In this study the overall GPS, as well as the GPS in the sagittal, coronal and transverse planes were calculated. All parameters were automatically extracted from the kinematic waveforms using custom-made MATLAB software (Mathworks 2017, Natick, MA, USA). Per subject, the value of each GA it parameter for each of three or more gait cycles were averaged.

Secondary outcome parameters were a set of discrete parameters that were selected based on clinical relevance. These were extracted from the continuous waveforms, or- ganized in gait cycles for kinematics and included mean pelvic tilt and rotation during the gait cycle, pelvic tilt, obliquity and rotation range of motion (ROM), maximum hip extension, maximum and minimum hip rotation, angle of the hip and knee at initial contact, timing and angle of maximum knee extension and flexion during stance and swing phase, respectively, knee flexion ROM and maximum ankle dorsiflexion during stance phase.

To address the first research question on the effects of MLS on gait in HSP children, means were calculated per gait parameter preoperatively and at short and medium-term post-operatively. Due to the majority of parameters having a non-normal distribution, differences in kinematic parameters preoperatively and between the follow-up moments were analysed using a Wilcoxon signed-rank test. To address the second question on the differences in outcome between HSP and $\mathrm{CP}$ children, the pre-operative and the medium-term FU change values were compared between HSP and CP groups us-

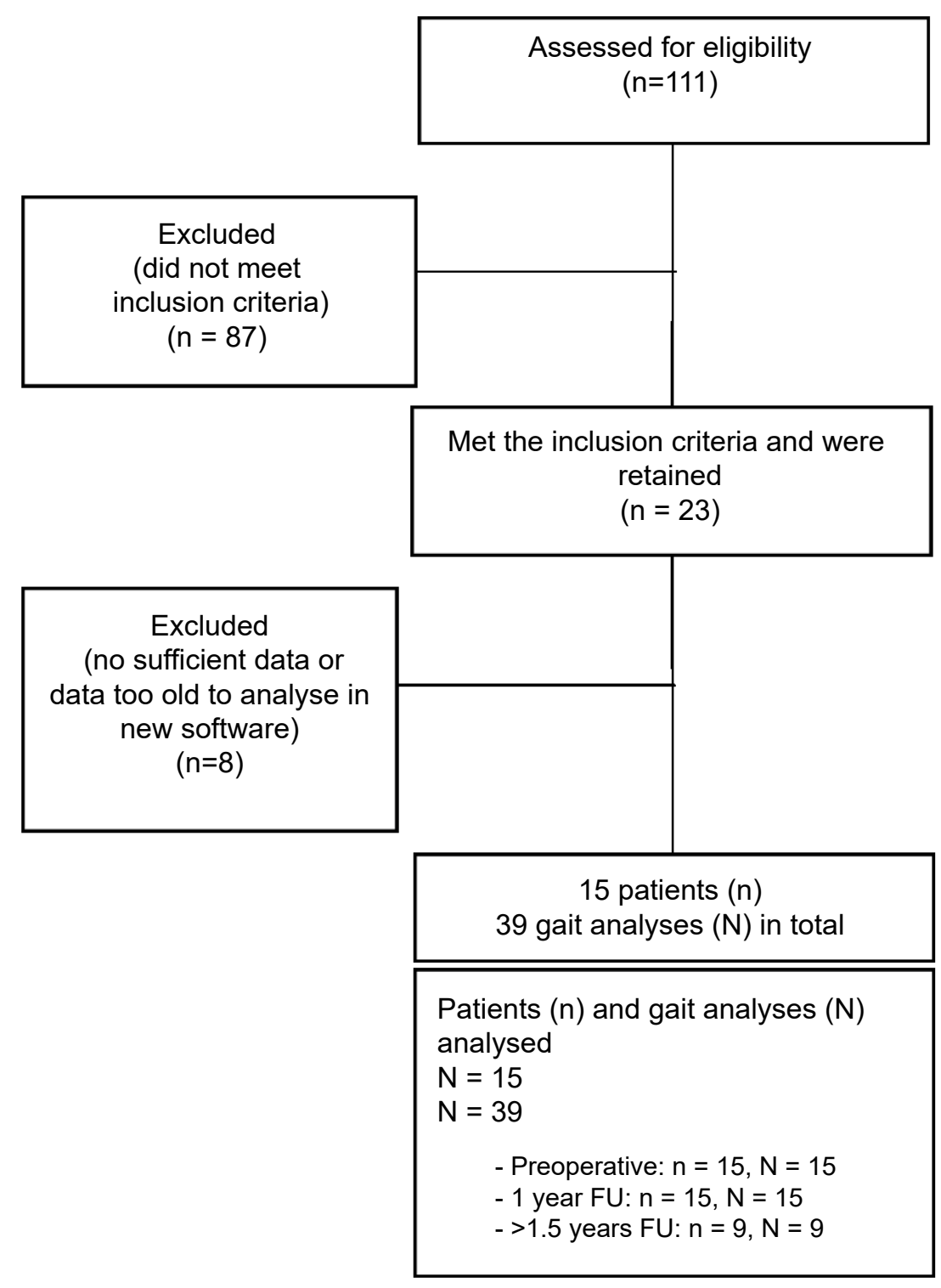

Figure 1: Flow-chart of selection procedure of children with HSP. 
Citation: Prinsen S, Buyck C, Beukelaer N, et al. (2020) The Effect of Multilevel Surgery on the Gait Pattern of Children with Hereditary Spastic Paraplegia, are there Differences in Outcome Compared to Those with Bilateral Spastic Cerebral Palsy?. J Orthop Surg Tech 3(2):179-190

ing Mann Whitney U-tests. To address the third research question on possible predictors for good outcome, the correlation between change in GPS pre-post and preoperative spatiotemporal and kinematic parameters and the post-operative gait parameters was explored using Spearman's correlation coefficients. SPSS statistics version 20.0 (SPSS Inc Chicago, USA) was used to perform the statistical analysis. To indicate the significance of all tests, an alpha level below 0.05 was used.

\section{Results}

One hundred and eleven patients with HSP were screened for eligibility and 15 patients were included (Figure 1). All 15 patients had gait analyses preoperative, with an average of 3.2 months (range 1-6 months) period prior to surgery and about 1 year after surgery, with an average interval of $11.6 \pm$ 2 months between surgery and the gait analysis. In 9 subjects, GA was also performed at middle-term FU (on average at 33.7 \pm 9 months post-surgery).

Seven patients had Botulinum toxin A-treatments and 2 patients had an ITB-pump, more than 6 months before MLS. Three patients had minor further surgery during the follow-up period and 4 patients received Botulinum toxin A-injections, but this at least 6 months before their follow-up gait analysis.

Nine patients with bilateral spastic CP who had undergone MLS, and who matched with the 9 HSP subjects with medium-term FU, were selected from the CMAL database. The characteristics of both groups can be found in (Table 1).

\section{What are the effects of MLS on the gait pattern of children with HSP at short-and medium-term follow up?}

Evolution of MAPS and GPS over time is shown in (Figure 2 and Figure 3 ) respectively. Overall GPS ( $p=0.021)$ and GPS in the sagittal plane ( $p=0.028$ ) improved significantly at medium-term FU, compared to the pre-surgery condition. At short-term, MAPS improved significantly for knee flexion ( $p=$ 0.006 ) and foot progression ( $p=0.027$ ) but had significantly poorer outcome for hip abduction $(p=0.020)$. There was a

Table 1: Characteristics of children pre-Multilevel Surgery (MLS) and details of the MLS operation.

\begin{tabular}{|c|c|c|c|}
\hline Characteristics & HSP (n = 15) & $\begin{array}{l}\text { HSP }(n=9) \\
>1.5 \text { yrs }\end{array}$ & $C P(n=9)$ \\
\hline GMFCS level I & 6 & 4 & 4 \\
\hline GMFCS level II & 6 & 4 & 5 \\
\hline GMFCS level III & 3 & 1 & 0 \\
\hline Male & 10 & 6 & 5 \\
\hline Female & 5 & 3 & 4 \\
\hline Mean [range] age at operation, years & $11.6[5.3-18.9]$ & $11.4[6.8-18.9]$ & $10.5[8.3-14.9]$ \\
\hline Mean weight pre-operative, kilograms & 37.13 & 37.7 & 30.43 \\
\hline Mean height pre-operative, meters & 1.4 & 1.41 & 1.34 \\
\hline \multicolumn{4}{|l|}{ Bony procedure } \\
\hline Femoral derotation osteotomy & 6 & 4 & 6 \\
\hline Femoral varisation osteotomy & 0 & 0 & 0 \\
\hline Femoral extension osteotomy & 1 & 0 & 1 \\
\hline Femoral derotation-varisation (DVO) & 5 & 3 & 0 \\
\hline Femoral derotation-extension & 3 & 2 & 1 \\
\hline Tibial derotation osteotomy & 5 & 3 & 6 \\
\hline Calcaneal lengthening osteotomy & 1 & 0 & 1 \\
\hline Patellar distalisation & 3 & 2 & 3 \\
\hline Talonavicular fusion & 1 & 0 & 1 \\
\hline \multicolumn{4}{|l|}{ Soft tissue procedure } \\
\hline Lengthening $\mathrm{m}$. psoas & 3 & 4 & 7 \\
\hline Lengthening Hamstrings & 3 & 3 & 7 \\
\hline Lengthening $\mathrm{m}$. gastrocnemius & 2 & 2 & 7 \\
\hline Lengtheningtib. posterior & 0 & 0 & 1 \\
\hline Lengthening Adductors & 3 & 1 & 1 \\
\hline Transfer $\mathrm{m}$. rectus femoris & 6 & 4 & 7 \\
\hline Botox & 9 & 5 & 0 \\
\hline
\end{tabular}

HSP: Hereditary Spastic Paraplegia; CP: Cerebral Palsy; GMFCS: Gross Motor Function Classification System. 


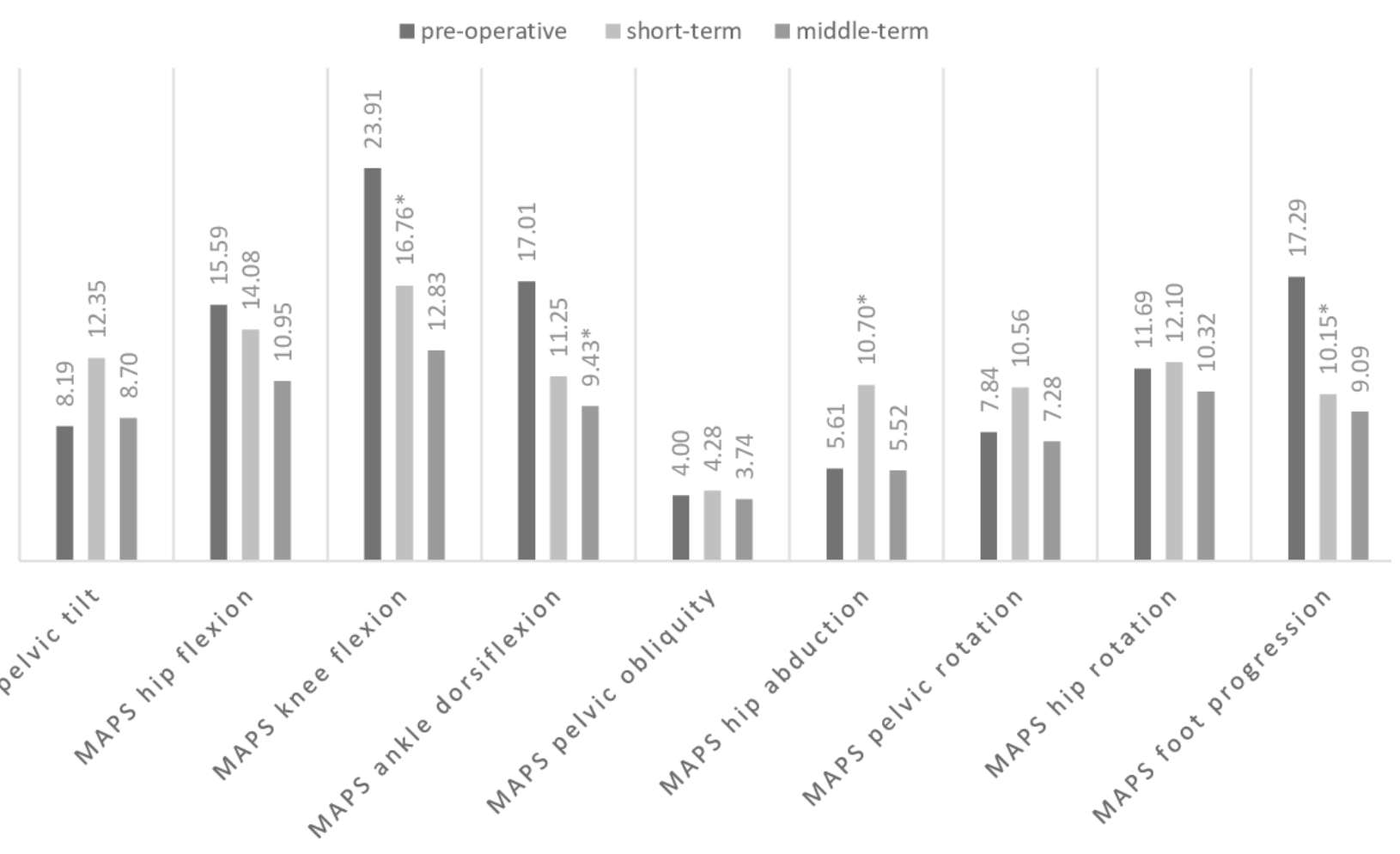

Figure 2: Root mean square (RMS) difference $\left({ }^{\circ}\right)$ of movement analysis profile score (MAPS) of HSP children.

${ }^{*}=\mathrm{P}<0.05$

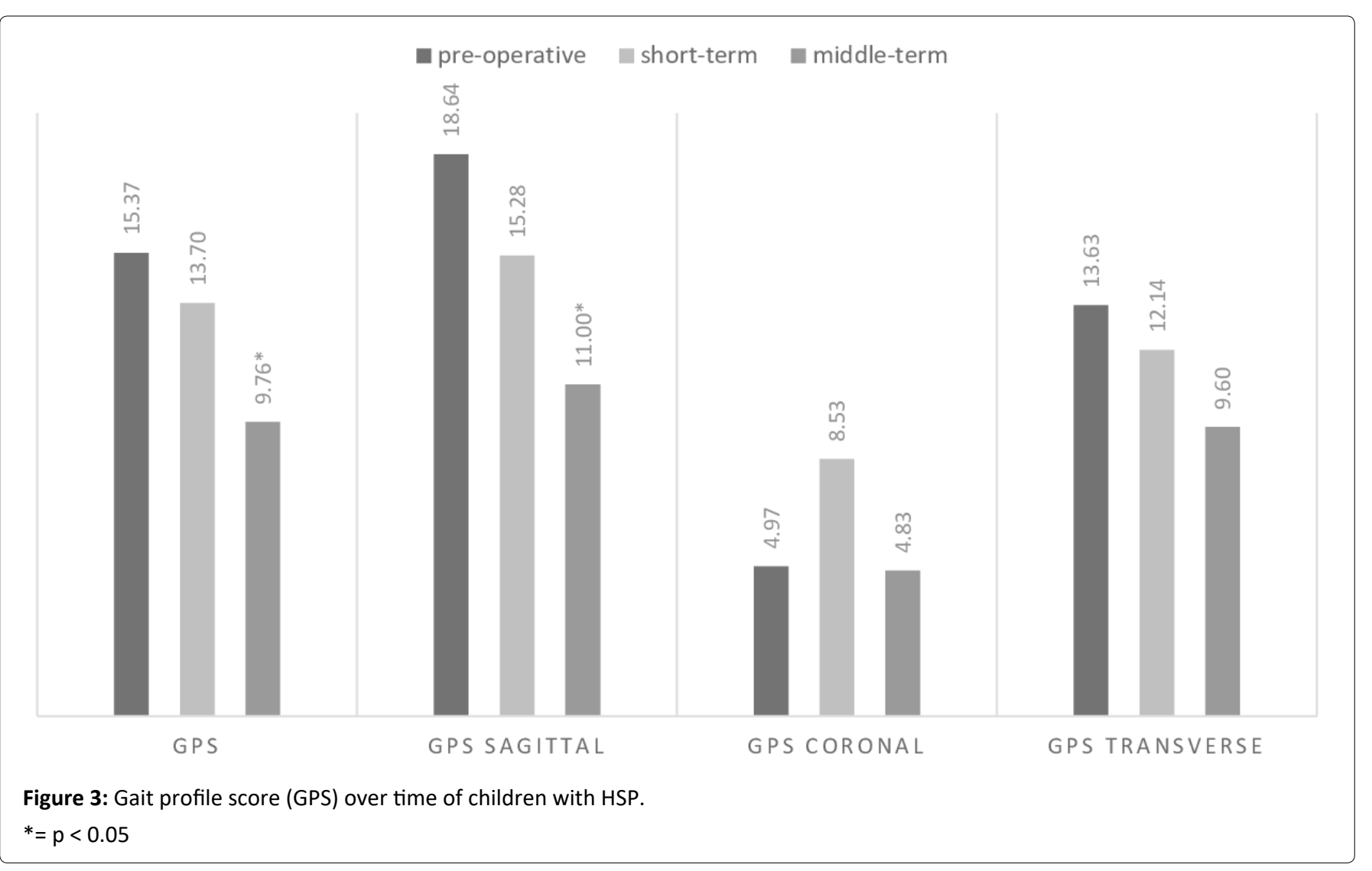


Citation: Prinsen S, Buyck C, Beukelaer N, et al. (2020) The Effect of Multilevel Surgery on the Gait Pattern of Children with Hereditary Spastic Paraplegia, are there Differences in Outcome Compared to Those with Bilateral Spastic Cerebral Palsy?. J Orthop Surg Tech 3(2):179-190

Table 2: Selected mean $( \pm 1 S D)$ of gait and kinematic parameters of pelvis, hip, knee and ankle for the group of children with HSP preoperative, after short-and middle-term FU.

\begin{tabular}{|c|c|c|c|c|c|c|}
\hline & \multicolumn{3}{|l|}{ Timing } & \multicolumn{3}{|l|}{ Statistic } \\
\hline & Pre-op & $<1.5$ yr FU & $>1.5 \mathrm{yr} F U$ & $<1.5 \mathrm{yr} F U$ & $>1.5 y r$ FU & $>1.5 y r$ FU \\
\hline & $\operatorname{HSP}(n=15)$ & $\operatorname{HSP}(n=15)$ & $\operatorname{HSP}(n=9)$ & vs. pre-op & vs. pre-op & vs. $<1.5$ yr FU \\
\hline & Mean \pm 1 SD & Mean $\pm 1 S D$ & Mean \pm 1 SD & $p$-value & $p$-value & $p$-value \\
\hline \multicolumn{7}{|l|}{ Gait measures } \\
\hline Cadence (1/s) & $1.89 \pm 0.38$ & $1.62 \pm 0.53$ & $1.81 \pm 0.37$ & $0.013^{*}$ & 0.375 & 0.678 \\
\hline Stride length (m) & $0.64 \pm 0.52$ & $0.57 \pm 0.41$ & $0.68 \pm 0.35$ & 0.064 & 0.953 & 0.441 \\
\hline Walking velocity $(\mathrm{m} / \mathrm{s})$ & $0.86 \pm 0.24$ & $0.68 \pm 0.29$ & $0.87 \pm 0.29$ & $0.016^{*}$ & 0.678 & 0.214 \\
\hline \multicolumn{7}{|l|}{ Pelvis } \\
\hline Mean pelvic tilt $\left({ }^{\circ}\right)$ & $17.79 \pm 5.29$ & $16.63 \pm 9.78$ & $19.60 \pm 3.69$ & 0.733 & 0.953 & 0.441 \\
\hline ROM pelvic tilt $\left({ }^{\circ}\right)$ & $10.16 \pm 3.67$ & $8.40 \pm 3.30$ & $8.60 \pm 3.02$ & $0.009^{*}$ & 0.515 & $0.021^{*}$ \\
\hline ROM pelvic obliquity $\left({ }^{\circ}\right)$ & $11.45 \pm 5.38$ & $8.69 \pm 3.06$ & $9.66 \pm 3.24$ & $0.047^{*}$ & 0.173 & 0.11 \\
\hline Mean pelvic rotation $\left({ }^{\circ}\right)$ & $0.10 \pm 0.39$ & $-0.19 \pm 0.17$ & $0.05 \pm 0.39$ & 0.865 & 0.594 & 0.441 \\
\hline ROM pelvic rotation $\left({ }^{\circ}\right)$ & $22.13 \pm 9.18$ & $20.35 \pm 7.65$ & $16.66 \pm 6.67$ & 0.427 & 0.11 & 0.214 \\
\hline \multicolumn{7}{|l|}{ Hip } \\
\hline Max hip extension during stance $\left({ }^{\circ}\right)$ & $5.39 \pm 15.67$ & $2.43 \pm 13.88$ & $0.12 \pm 11.06$ & 0.394 & 0.767 & 0.953 \\
\hline Max hip rotation $\left({ }^{\circ}\right)$ & $18.53 \pm 6.27$ & $7.09 \pm 10.22$ & $9.61 \pm 6.49$ & $0.003^{* *}$ & $0.008^{*}$ & 0.594 \\
\hline Min hip rotation $\left({ }^{\circ}\right)$ & $-4.01 \pm 7.64$ & $-15.31 \pm 8.57$ & $-15.55 \pm 7.56$ & $0.006^{*}$ & $0.011^{*}$ & 0.859 \\
\hline Angle at initial contact $\left({ }^{\circ}\right)$ & $47.02 \pm 8.38$ & $40.48 \pm 11.03$ & $41.17 \pm 8.08$ & 0.053 & 0.11 & 0.26 \\
\hline \multicolumn{7}{|l|}{ Knee } \\
\hline Max knee extension during stance $\left({ }^{\circ}\right)$ & $19.79 \pm 22.21$ & $3.28 \pm 9.66$ & $4.55 \pm 7.23$ & $0.015^{*}$ & 0.26 & 0.953 \\
\hline Timing max knee extension (\%) & $31.65 \pm 9.33$ & $32.59 \pm 9.64$ & $32.68 \pm 8.29$ & 0.955 & 0.515 & 0.515 \\
\hline Max knee flexion during swing $\left({ }^{\circ}\right)$ & $62.12 \pm 9.49$ & $54.13 \pm 7.70$ & $58.19 \pm 8.68$ & $0.020^{*}$ & 0.374 & 0.314 \\
\hline Timing max knee flexion (\%) & $80.10 \pm 4.72$ & $79.03 \pm 5.66$ & $76.10 \pm 3.58$ & 0.363 & 0.214 & 0.594 \\
\hline ROM knee flexion $\left({ }^{\circ}\right)$ & $42.33 \pm 21.32$ & $50.85 \pm 13.39$ & $53.64 \pm 9.14$ & 0.125 & 0.374 & 0.953 \\
\hline Angle at initial contact $\left({ }^{\circ}\right)$ & $37.02 \pm 16.35$ & $19.83 \pm 11.63$ & $20.36 \pm 9.55$ & $0.002^{* *}$ & 0.051 & 0.678 \\
\hline \multicolumn{7}{|l|}{ Ankle } \\
\hline Max ankle dorsiflexion during stance $\left({ }^{\circ}\right)$ & $12.58 \pm 17.50$ & $13.80 \pm 6.59$ & $14.74 \pm 6.94$ & 0.865 & 0.859 & 0.953 \\
\hline
\end{tabular}

${ }^{*} \mathrm{p}<0.05 ;{ }^{* *} \mathrm{p}<0.005$; ROM: Range of Motion; max: Maximal; min: Minimal.

significant further improvement in MAPS of ankle dorsiflexion $(p=0.008)$ and GPS in the sagittal plane $(p=0.038)$ between short- and medium-term FU.

The means of overall gait and kinematic parameters are shown in (Table 2). Of the spatial-temporal parameters, cadence and walking velocity decreased significantly at shortterm in the HSP group. However, at medium-term, these values returned to the starting values. Minimal and maximal hip rotation showed significant changes at short-term and these improvements remained stable over time. At medium-term FU, pelvic tilt ROM showed a further significant decrease compared to short-term FU. Maximum knee flexion during swing was significantly lower at short-term FU. ROM of pelvic tilt, ROM of pelvic obliquity, maximum knee extension during stance and the hip and knee angles at initial contact showed significant improvements in the short-term, but were no longer significant at medium-term FU.

Are there differences in outcome post-MLS in HSP children compared to those with CP?

The selected means of overall gait and kinematic param- eters for the children with CP are shown in (Table 3). GPS significantly improved in the medium-term for children with CP. The comparison of pre-operative values is shown in (Table 4): Only pelvic rotation ROM ( $p=0.022)$, maximal knee flexion during swing $(p=0.029)$, knee flexion ROM $(p=0.025)$ and the MAPS hip abduction ( $p=0.041$ ) were significantly lower for children with $\mathrm{CP}$, compared to children with HSP.

The comparison of change values pre vs. post-operatively at medium-term FU for the two groups are shown in (Table 5). Most postoperative changes in gait were similar for $C P$ and HSP children; only mean pelvic rotation increased significantly more for children with $\mathrm{CP}$ at medium-term FU than for children with HSP.

\section{Are there possible predictors for a good out- come after MLS in children with HSP?}

Scatter plots of good $(r \geq 0.50)$ correlations between pre-operative parameters and change in GPS at short-term FU are shown in (Figure 4) and for medium-term FU in (Figure 5). The following pre-operative parameters correlated significantly with change in GPS compared to pre-operatively at 
Citation: Prinsen S, Buyck C, Beukelaer N, et al. (2020) The Effect of Multilevel Surgery on the Gait Pattern of Children with Hereditary Spastic Paraplegia, are there Differences in Outcome Compared to Those with Bilateral Spastic Cerebral Palsy?. J Orthop Surg Tech 3(2):179-190

Table 3: Selected mean $( \pm 1 S D)$ of gait and kinematic parameters of pelvis, hip, knee and ankle for the group of children with CP pre-operative and at medium-term FU.

\begin{tabular}{|c|c|c|c|}
\hline & Timing & & Statistics \\
\hline & pre-op & $>1.5 \mathrm{yr}$ FU & \\
\hline & $C P(n=9)$ & $C P(n=9)$ & \\
\hline & Mean \pm 1SD & Mean \pm 1SD & $p$-value \\
\hline cadence $(1 / \mathrm{s})$ & $2.04 \pm 0.33$ & $1.82 \pm 0.30$ & $\leq 0.001^{*}$ \\
\hline stride length (m) & $0.43 \pm 0.09$ & $0.42 \pm 0.10$ & 0.553 \\
\hline walking velocity $(\mathrm{m} / \mathrm{s})$ & $0.88 \pm 0.21$ & $0.78 \pm 0.22$ & $0.028^{*}$ \\
\hline \multicolumn{4}{|l|}{ Pelvis } \\
\hline Mean pelvic tilt $\left({ }^{\circ}\right)$ & $17.82 \pm 4.53$ & $17.58 \pm 4.31$ & 0.915 \\
\hline ROM pelvic tilt $\left({ }^{\circ}\right)$ & $9.79 \pm 2.64$ & $7.00 \pm 1.74$ & $0.011^{*}$ \\
\hline ROM pelvic obliquity $\left({ }^{\circ}\right)$ & $11.22 \pm 4.58$ & $11.05 \pm 1.96$ & 0.89 \\
\hline Mean pelvic rotation $\left({ }^{\circ}\right)$ & $-0.29 \pm 0.65$ & $0.22 \pm 0.64$ & 0.066 \\
\hline ROM pelvic rotation $\left({ }^{\circ}\right)$ & $15.29 \pm 5.49$ & $18.38 \pm 3.51$ & 0.087 \\
\hline \multicolumn{4}{|l|}{ Hip } \\
\hline max hip extension during stance phase $\left({ }^{\circ}\right)$ & $4.34 \pm 3.16$ & $4.50 \pm 355$ & 0.878 \\
\hline Max hip rotation $\left({ }^{\circ}\right)$ & $18.83 \pm 6.67$ & $12.89 \pm 8.84$ & $0.025^{*}$ \\
\hline min hip rotation $\left({ }^{\circ}\right)$ & $-0.75 \pm 6.01$ & $-10.89 \pm 10.59$ & $0.010^{*}$ \\
\hline \multicolumn{4}{|l|}{ Knee } \\
\hline Max knee extension in stance phase $\left({ }^{\circ}\right)$ & $18.33 \pm 8.45$ & $10.12 \pm 7.15$ & 0.075 \\
\hline Timing max knee extension (\%) & $29.47 \pm 5.24$ & $36.17 \pm 5.32$ & $0.026^{*}$ \\
\hline Max knee flexion during swing $\left({ }^{\circ}\right)$ & $59.87 \pm 5.95$ & $62.13 \pm 8.24$ & 0.582 \\
\hline timing max knee flexion during swing (\%) & $77.16 \pm 4.36$ & $75.47 \pm 4.36$ & 0.088 \\
\hline ROM knee flexion $\left({ }^{\circ}\right)$ & $41.54 \pm 10.08$ & $52.01 \pm 9.25$ & 0.057 \\
\hline Angle at initial contact $\left({ }^{\circ}\right)$ & $31.99 \pm 8.09$ & $23.22 \pm 7.45$ & $0.011^{*}$ \\
\hline \multicolumn{4}{|l|}{ Ankle } \\
\hline Max ankle dorsiflexion during stance phase $\left({ }^{\circ}\right)$ & $14.36 \pm 7.31$ & $18.13 \pm 2.94$ & 0.173 \\
\hline \multicolumn{4}{|l|}{ MAPS and GPS } \\
\hline MAPS pelvic tilt $\left({ }^{\circ}\right)$ & $8.10 \pm 3.28$ & $6.99 \pm 3.60$ & 0.555 \\
\hline MAPS hip flexion $\left({ }^{\circ}\right)$ & $13.55 \pm 2.13$ & $11.38 \pm 2.81$ & 0.21 \\
\hline MAPS knee flexion $\left({ }^{\circ}\right)$ & $18.34 \pm 5.29$ & $14.31 \pm 5.78$ & 0.059 \\
\hline MAPS ankle dorsiflexion $\left({ }^{\circ}\right)$ & $10.24 \pm 3.43$ & $9.98 \pm 2.22$ & 0.853 \\
\hline MAPS pelvic obliquity $\left({ }^{\circ}\right)$ & $3.36 \pm 1.23$ & $3.25 \pm 0.72$ & 0.797 \\
\hline MAPS hip abduction $\left({ }^{\circ}\right)$ & $4.91 \pm 1.14$ & $4.90 \pm 0.94$ & 0.985 \\
\hline MAPS pelvic rotation $\left({ }^{\circ}\right)$ & $6.24 \pm 3.02$ & $6.52 \pm 2.68$ & 0.797 \\
\hline MAPS hip rotation $\left({ }^{\circ}\right)$ & $12.88 \pm 3.11$ & $10.86 \pm 4.08$ & 0.187 \\
\hline MAPS foot progression $\left({ }^{\circ}\right)$ & $14.30 \pm 312.58$ & $8.92 \pm 3.30$ & 0.314 \\
\hline GPS $\left({ }^{\circ}\right)$ & $12.35 \pm 2.80$ & $9.70 \pm 2.20$ & $0.021^{*}$ \\
\hline GPS sagittal $\left({ }^{\circ}\right)$ & $13.56 \pm 2.60$ & $11.47 \pm 2.58$ & 0.84 \\
\hline GPS coronal $\left({ }^{\circ}\right)$ & $4.30 \pm 1.00$ & $4.21 \pm 0.80$ & 0.802 \\
\hline GPS transverse $\left({ }^{\circ}\right)$ & $12.77 \pm 6.45$ & $9.30 \pm 3.13$ & 0.11 \\
\hline
\end{tabular}

${ }^{*} \mathrm{P}<$ 0.05; ROM: Range of Motion; max: Maximal; min: Minimal; MAPS: Movement Analysis Profile Score; GPS: Gait Profile Score. 
Citation: Prinsen S, Buyck C, Beukelaer N, et al. (2020) The Effect of Multilevel Surgery on the Gait Pattern of Children with Hereditary Spastic Paraplegia, are there Differences in Outcome Compared to Those with Bilateral Spastic Cerebral Palsy?. J Orthop Surg Tech 3(2):179-190

Table 4: Mean $( \pm 1 S D)$ values of gait measures and kinematic parameters from a group of children with HSP compared with children with CP pre-operatively.

\begin{tabular}{|c|c|c|c|}
\hline & Timing & & Statistics \\
\hline & $\operatorname{HSP}(n=9)$ & $C P(n=9)$ & \\
\hline & Pre-op & Pre-op & $p$-value \\
\hline Cadence (1/s) & $1.96 \pm 0.29$ & $2.04 \pm 0.33$ & 0.981 \\
\hline Stride length (m) & $0.77 \pm 0.64$ & $0.43 \pm 0.09$ & 0.387 \\
\hline Walking velocity $(\mathrm{m} / \mathrm{s})$ & $0.90 \pm 0.25$ & $0.88 \pm 0.21$ & 0.666 \\
\hline ROM pelvic tilt $\left({ }^{\circ}\right)$ & $9.10 \pm 1.83$ & $9.79 \pm 2.64$ & 0.051 \\
\hline Mean pelvic tilt $\left({ }^{\circ}\right)$ & $19.32 \pm 4.76$ & $17.82 \pm 4.53$ & 0.625 \\
\hline ROM pelvic obliquity $\left({ }^{\circ}\right)$ & $12.04 \pm 4.39$ & $11.22 \pm 4.58$ & 0.671 \\
\hline Mean pelvic rotation $\left({ }^{\circ}\right)$ & $-0.01 \pm 0.35$ & $-0.29 \pm 0.65$ & 0.113 \\
\hline ROM pelvic rotation $\left({ }^{\circ}\right)$ & $22.97 \pm 10.55$ & $15.29 \pm 5.49$ & $0.022^{*}$ \\
\hline Max hip extension during stance $\left({ }^{\circ}\right)$ & $3.47 \pm 17.33$ & $4.34 \pm 3.16$ & 0.19 \\
\hline Max hip rotation $\left({ }^{\circ}\right)$ & $20.67 \pm 5.25$ & $18.33 \pm 8.45$ & 0.491 \\
\hline Min hip rotation $\left({ }^{\circ}\right)$ & $-2.19 \pm 5.87$ & $-0.75 \pm 9.97$ & 0.598 \\
\hline Max knee extension during stance $\left({ }^{\circ}\right)$ & $15.21 \pm 22.31$ & $18.33 \pm 9.56$ & 0.054 \\
\hline Timing max knee extension (\%) & $28.31 \pm 9.96$ & $29.47 \pm 5.24$ & 0.863 \\
\hline Max knee flexion during swing $\left({ }^{\circ}\right)$ & $60.58 \pm 10.43$ & $59.87 \pm 5.95$ & $0.029^{*}$ \\
\hline Timing max knee flexion during swing (\%) & $78.39 \pm 4.22$ & $77.16 \pm 4.36$ & 0.925 \\
\hline ROM knee flexion $\left({ }^{\circ}\right)$ & $45.37 \pm 24.44$ & $41.54 \pm 10.08$ & $0.025^{*}$ \\
\hline knee angle at initial contact $\left({ }^{\circ}\right)$ & $32.85 \pm 16.03$ & $31.99 \pm 8.09$ & 0.231 \\
\hline max ankle dorsiflexion during stance $\left({ }^{\circ}\right)$ & $14.40 \pm 11.30$ & $14.36 \pm 7.31$ & 0.474 \\
\hline MAPS pelvic tilt $\left({ }^{\circ}\right)$ & $8.95 \pm 3.47$ & $8.10 \pm 3.28$ & 0.584 \\
\hline MAPS hip flexion $\left({ }^{\circ}\right)$ & $14.67 \pm 11.12$ & $13.55 \pm 2.13$ & 0.19 \\
\hline MAPS knee flexion $\left({ }^{\circ}\right)$ & $21.49 \pm 12.13$ & $18.34 \pm 5.29$ & 1 \\
\hline MAPS ankle dorsiflexion $\left({ }^{\circ}\right)$ & $11.21 \pm 6.32$ & $10.24 \pm 3.34$ & 1 \\
\hline MAPS pelvic obliquity $\left({ }^{\circ}\right)$ & $3.82 \pm 1.14$ & $3.36 \pm 1.23$ & 0.917 \\
\hline MAPS hip abduction $\left({ }^{\circ}\right)$ & $5.40 \pm 1.76$ & $4.91 \pm 1.14$ & $0.041^{*}$ \\
\hline MAPS pelvic rotation $\left({ }^{\circ}\right)$ & $7.57 \pm 2.56$ & $6.24 \pm 3.02$ & 0.912 \\
\hline MAPS hip rotation $\left({ }^{\circ}\right)$ & $12.49 \pm 3.49$ & $12.88 \pm 3.11$ & 0.775 \\
\hline MAPS foot progression $\left({ }^{\circ}\right)$ & $15.18 \pm 4.69$ & $14.30 \pm 12.58$ & 0.19 \\
\hline GPS & $13.47 \pm 4.29$ & $12.35 \pm 2.80$ & 0.73 \\
\hline GPS sagittal & $15.60 \pm 7.77$ & $13.56 \pm 2.60$ & 0.931 \\
\hline GPS coronal & $4.77 \pm 1.33$ & $4.30 \pm 1.00$ & 0.117 \\
\hline GPS transverse & $12.70 \pm 2.78$ & $12.77 \pm 6.45$ & 0.546 \\
\hline
\end{tabular}

" $p$ < 0.05; ROM: Range of Motion; max: maximal; min; minimal; MAPS: Movement Analysis Profile Score; GPS: Gait Profile Score.

Table 5: Mean $( \pm 1 S D)$ change in gait measures and kinematic parameters between pre- and medium term follow-up for a group of children with HSP and a group of children with CP.

\begin{tabular}{|l|l|l|l|}
\hline \multirow{2}{*}{} & Timing & Statistics & \\
\cline { 2 - 4 } & HSP $(\mathrm{n}=9)$ & CP $(\mathrm{n}=9)$ & \\
\cline { 2 - 4 } & {$[2-4 \mathrm{yr}]$ post-op-pre-op } & {$[2-3$ yr] post-op-pre- op } & p-value \\
\hline Cadence $(1 / \mathrm{s})$ & $-0.15 \pm 0.34$ & $-0.22 \pm 0.10$ & 0.056 \\
\hline Stride length $(\mathrm{m})$ & $-0.09 \pm 0.34$ & $-0.01 \pm 0.06$ & 0.546 \\
\hline Walking velocity $(\mathrm{m} / \mathrm{s})$ & $-0.03 \pm 0.34$ & $-0.09 \pm 0.08$ & 0.051 \\
\hline ROM pelvic tilt $\left(^{\circ}\right)$ & $-0.50 \pm 2.92$ & $-2.78 \pm 2.39$ & 0.965 \\
\hline
\end{tabular}


Citation: Prinsen S, Buyck C, Beukelaer N, et al. (2020) The Effect of Multilevel Surgery on the Gait Pattern of Children with Hereditary Spastic Paraplegia, are there Differences in Outcome Compared to Those with Bilateral Spastic Cerebral Palsy?. J Orthop Surg Tech 3(2):179-190

\begin{tabular}{|c|c|c|c|}
\hline Mean pelvic tilt $\left({ }^{\circ}\right)$ & $0.28 \pm 6.86$ & $-0.24 \pm 6.09$ & 0.997 \\
\hline ROM pelvic obliquity $\left(^{\circ}\right)$ & $-2.38 \pm 4.82$ & $-0.18 \pm 5.03$ & 0.993 \\
\hline Mean pelvic rotation $\left({ }^{\circ}\right)$ & $0.06 \pm 0.30$ & $0.51 \pm 0.90$ & $0.028^{*}$ \\
\hline ROM pelvic rotation $\left({ }^{\circ}\right)$ & $-6.30 \pm 10.97$ & $3.09 \pm 4.47$ & 0.083 \\
\hline Max hip extension during stance $\left({ }^{\circ}\right)$ & $-3.35 \pm 15.12$ & $0.16 \pm 2.87$ & 1 \\
\hline Max hip rotation $\left({ }^{\circ}\right)$ & $-11.07 \pm 7.62$ & $-5.94 \pm 6.08$ & 0.639 \\
\hline Min hip rotation $\left({ }^{\circ}\right)$ & $-13.36 \pm 8.57$ & $-10.14 \pm 8.52$ & 0.549 \\
\hline Max knee extension during stance $\left({ }^{\circ}\right)$ & $-10.66 \pm 20.16$ & $-8.22 \pm 11.34$ & 0.093 \\
\hline Timing max knee extension (\%) & $4.37 \pm 13.06$ & $6.70 \pm 6.96$ & 0.24 \\
\hline Max knee flexion during swing $\left({ }^{\circ}\right)$ & $-2.39 \pm 7.33$ & $2.26 \pm 11.14$ & 0.466 \\
\hline Timing max knee flexion during swing (\%) & $-2.30 \pm 4.48$ & $-1.68 \pm 2.45$ & 0.863 \\
\hline ROM knee flexion $\left({ }^{\circ}\right)$ & $8.27 \pm 18.98$ & $10.47 \pm 13.33$ & 0.305 \\
\hline knee angle at initial contact $\left({ }^{\circ}\right)$ & $-12.49 \pm 16.25$ & $-8.77 \pm 7.60$ & 0.127 \\
\hline max ankle dorsiflexion during stance $\left({ }^{\circ}\right)$ & $0.33 \pm 6.26$ & $3.77 \pm 7.42$ & 0.907 \\
\hline MAPS pelvic tilt $\left({ }^{\circ}\right)$ & $-0.25 \pm 5.28$ & $-1.10 \pm 5.07$ & 0.701 \\
\hline MAPS hip flexion $\left({ }^{\circ}\right)$ & $-3.71 \pm 9.91$ & $-2.17 \pm 4.51$ & 0.365 \\
\hline MAPS knee flexion $\left({ }^{\circ}\right)$ & $-8.66 \pm 12.98$ & $-4.03 \pm 5.17$ & 0.124 \\
\hline MAPS ankle dorsiflexion $\left({ }^{\circ}\right)$ & $-1.78 \pm 4.13$ & $-0.26 \pm 3.81$ & 0.856 \\
\hline MAPS pelvic obliquity $\left({ }^{\circ}\right)$ & $-0.08 \pm 1.81$ & $-0.11 \pm 1.16$ & 0.126 \\
\hline MAPS hip abduction $\left({ }^{\circ}\right)$ & $0.12 \pm 1.77$ & $-0.01 \pm 1.52$ & 0.631 \\
\hline MAPS pelvic rotation $\left({ }^{\circ}\right)$ & $-0.29 \pm 2.90$ & $0.28 \pm 2.94$ & 0.677 \\
\hline MAPS hip rotation $\left({ }^{\circ}\right)$ & $-2.18 \pm 5.02$ & $-2.02 \pm 3.75$ & 0.503 \\
\hline MAPS foot progression $\left({ }^{\circ}\right)$ & $-6.08 \pm 7.37$ & $-5.38 \pm 12.83$ & 0.416 \\
\hline GPS & $-3.71 \pm 4.25$ & $-2.65 \pm 3.08$ & 0.775 \\
\hline GPS sagittal & $-4.60 \pm 6.91$ & $-2.09 \pm 2.94$ & 0.419 \\
\hline GPS coronal & $0.07 \pm 1.30$ & $-0.09 \pm 1.20$ & 0.765 \\
\hline GPS transverse & $-3.10 \pm 4.65$ & $-3.46 \pm 6.35$ & 0.45 \\
\hline
\end{tabular}

" $\mathrm{p}$ < 0.05; ROM: Range of Motion, max: Maximal, min: Minimal, MAPS: Movement Analysis Profile Score, GPS: Gait Profile Score.

Note: negative values indicate a lower value at FU than pre-operatively. This can both mean an improvement or a deterioration, depending on the variable.
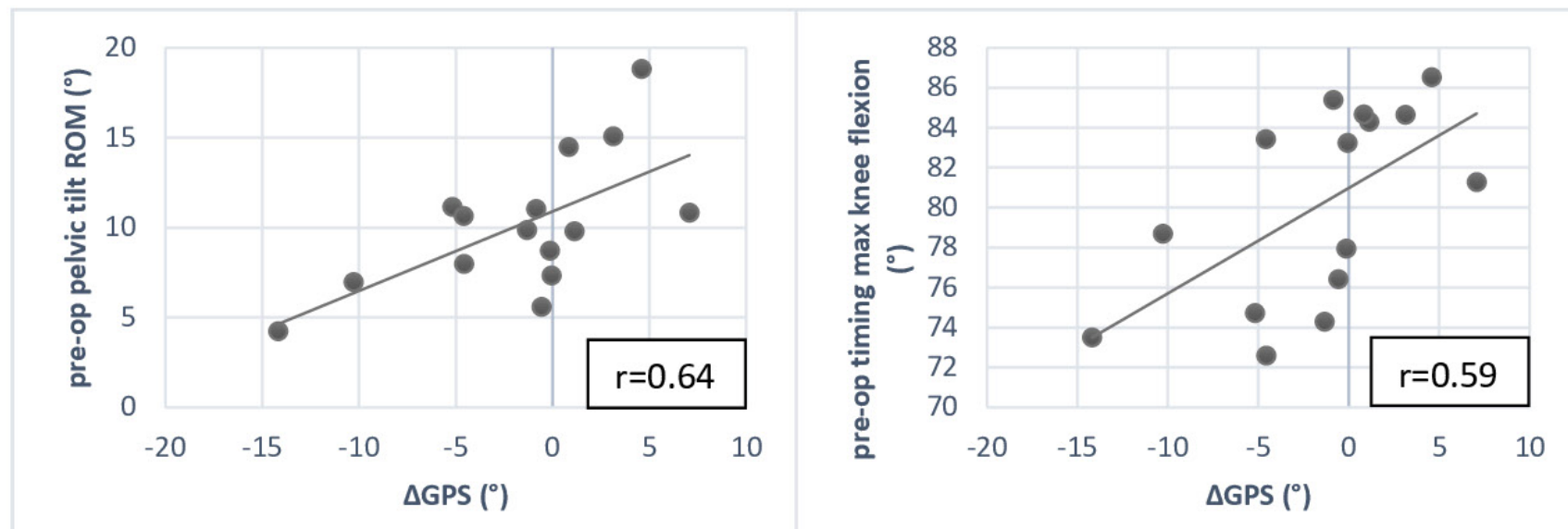

Figure 4: Scatterplots of good $(r \geq 0.50)$ correlations between pre-operative parameters and change in GPS at short-term FU. Pre-op, pre-operative; ROM: Range of Motion; $\Delta$, change; max: Maximal Note: negative values for change in GPS indicate a lower value at short-term FU than pre-operatively. 

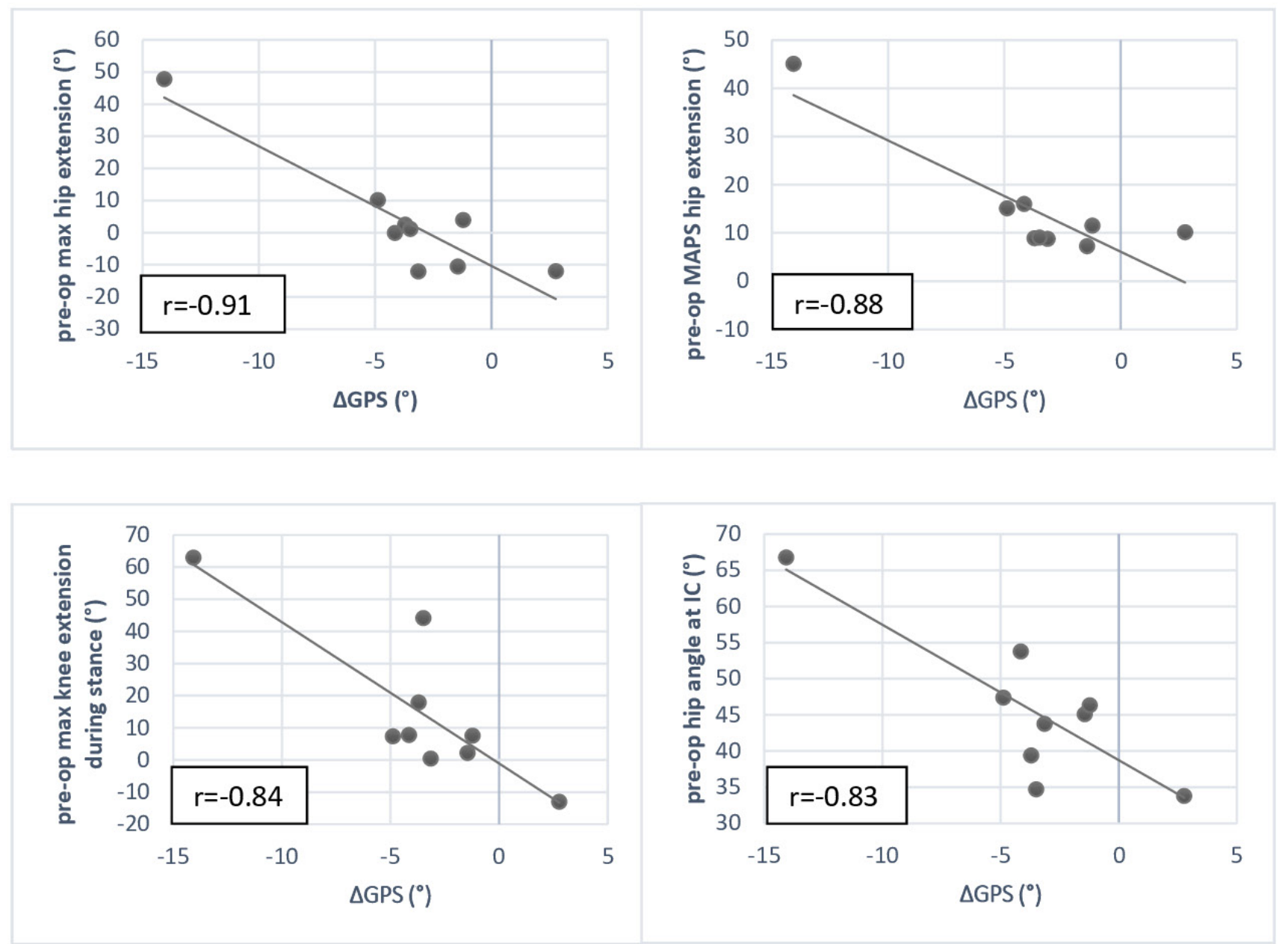

Figure 5: Scatterplots of significant correlations between pre-operative parameters and change in GPS at medium-term FU.

Pre-op, pre-operative; ROM: Range of Motion; $\Delta$, change; max: Maximal, IC: Initial Contact

Note: negative values for change in GPS indicate a lower value at short-term FU than pre-operatively.

short-term: Pelvic tilt ROM ( $r=0.64, p=0.011)$ and timing where maximum knee flexion occurred $(r=0.59, p=0.020)$. The parameters that correlated with change in GPS at medium-term were maximal hip extension during stance $(r=-0.91$, $p=0.001)$, hip angle at initial contact $(r=-0.83, p=0.005)$, knee angle at initial contact $(r=-0.83, p=0.002)$, maximal knee extension during stance $(r=-0.84, p=0.005)$, knee flexion ROM ( $r=0.71, p=0.032)$, GPS in the sagittal plane $(r=$ $-0.78, p=0.013)$, stride length $(r=0.76, p=0.017)$, MAPS of hip flexion $(r=-0.88, p=0.002)$, MAPS for knee flexion ( $r=$ $-0.76, p=0.018)$, GPS $(r=-0.76, p=0.017)$.

\section{Discussion}

This retrospective cohort study examined the short-and middle-term effects of MLS on the gait of children with HSP and compared them to the effects of MLS on a matched group of children with bilateral spastic CP. Post-surgery, compared to the pre-surgery condition, an overall improvement of gait was found, demonstrated by a decline in GPS for overall score and in GPS in the sagittal plane. This improvement was three to four times the minimal clinically important difference, which is 1.6 degrees for GPS [17] and this was comparable to the improvement of children with $\mathrm{CP}$.
This is the first study to document the effect of MLS on the gait of children with HSP. As the pathology is rare and heterogeneous, the CMAL database included only a small sample size of 15 children with HSP. For the comparison between HSP and $\mathrm{CP}$, at medium-term after surgery, only 9 patients could be compared. Hence, the results should be interpreted with caution. The design of this study is retrospective, therefore not every subject had a 3DGA at every or the exact same FU moments. Furthermore, while the group of $\mathrm{CP}$ patients was matched as good as possible for type of MLS, the group of CP patients received more soft tissue procedures than the HSP group. Also, there were some children in both groups that required other procedures, mostly botulinum toxin A-injections, during the follow up period. Furthermore, this study only gives us information about gait, not on gross motor function. Dequeker, et al. showed that the impact of MLS on CP children is more pronounced for functional mobility than for self-care [18].

\section{What are the effects of MLS on the gait pattern of children with HSP in short-and middle term follow up?}


Citation: Prinsen S, Buyck C, Beukelaer N, et al. (2020) The Effect of Multilevel Surgery on the Gait Pattern of Children with Hereditary Spastic Paraplegia, are there Differences in Outcome Compared to Those with Bilateral Spastic Cerebral Palsy?. J Orthop Surg Tech 3(2):179-190

A strong improvement of gait was documented after MLS. To fully interpret the results of surgery, we have to understand the natural evolution of children with HSP. Gait will deteriorate over time due to increasing weight and muscle spasticity [19] Van Campenhout, et al. previously found that patients with HSP presented with increased GPS, reduced walking speed and cadence and increased anterior pelvic tilt at follow-up [19].

In children with HSP we observed a significant decrease in ROM (range of motion) for pelvic tilt at short-and middle term follow-up after MLS. Both groups were characterised by increased pelvic tilt. The averaged sagittal position of the pelvic tilt stayed more or less stable over time in the children with $\mathrm{CP}$. Anterior pelvic tilt is a typical feature in children with HSP and CP (due to spastic hip flexors and weak hip extensors).

Lamberts, et al. concluded from their systematic review as that the long-term effects after MLS in children with CP were decreased pelvic tilt position and ROM [8]. The other kinematic parameters that significantly improved post-operatively in most of the studies included: Knee ROM, angle of the knee at initial contact, knee extension in stance, mean hip rotation and angle of foot progression. Similarly, in the current study, the knees of children with HSP presented less flexion at initial contact in the short-term, indicating a better knee-extension post-operatively. Surprisingly, the current sample of children with HSP did not maintain the prolonged knee hyperextension during stance phase over time, that could differ their gait from the gait of CP children $[5,6]$.

Cadence and walking speed of the children with HSP in our study decreased significantly one year after MLS. However, these differences disappeared at middle-term FU and there was a trend towards increasing stride length and walking velocity. We hypothesized that middle-term outcome are better than short-term results in children with HSP because they need more time to reach plateau due to weakness which is often significant in children with HSP. In comparison to the children with HSP, the cadence and walking velocity of the children with CP significantly decreased at middle-term. Overall, we can conclude that gait improves following MLS.

\section{Are there differences in outcome post-MLS in HSP children compared to those with CP?}

One of the most frequently performed bony procedures as part of MLS is a femoral derotation osteotomy. Indeed, 6 children with HSP from the current study received this bony correction and another 5 children had a derotation together with a varisation of the proximal femur to improve hip coverage. Thereby, the internal and external rotation of the hip of children with HSP improved at short- and middle-term. The same effect was noticed in the CP group, where also 6 out of 9 children had a derotation and 1 patient had a derotation together with an extension osteotomy of the femur. Saglam, et al. studied this particular procedure as part of MLS in CP and found similar effects. They found the most prominent effects on the hip in the transverse plane and the angle of foot progression [20]. An important side note to this is that pelvic rotation ROM decreased, although not significant, for the current group of children with HSP, but increased for children with $\mathrm{CP}$ at middle-term FU. Decrease in pelvic rotation ROM is an expected outcome when these children have femoral derotation as part of their MLS. Post-operative internal hip rotation was identified as a predictor for residual pelvic rotation after MLS in a study by Chung, et al. [21].

Although internal hip rotation decreased for both of the groups, it decreased more for children with HSP than those with $\mathrm{CP}$, but this change was also found not to be significant. Likewise, in a study by Rutz, et al. it was shown that pelvic rotation for children with $\mathrm{CP}$ decreased in the short-term post MLS, but in contrary to the current study sample, it increased again in the middle-term to be even larger than pre-operatively [22].

We can conclude that since the same principles are applied in surgical treatment for CP and HSP, including the concept of correction of the bony alignment, SMLs resulted in an equal improvement of gait in HSP and CP children.

\section{Are there possible predictors for a good out- come after MLS in children with HSP?}

Children with the highest overall GPS and GPS in the sagittal plane benefit the most from MLS, with largest improvement in GPS values at middle-term FU. Also for the hip, as well as for the knee joint, significant correlations were found. In clinical terms, for patients with severe preoperative pathology, we found the best postoperative improvement. Furthermore, the lower the knee flexion ROM pre-operatively (more severe stiff-knee pattern, possibly due to spasticity of the rectus femoris), the more GPS decreased at middle-term FU. All these results also suggest that patients who presented with increased flexion gait patterns in the hip and knee joint pre-operatively were likely to show more progress in their gait pattern post-MLS. Another pre-operative value that could influence the outcome is the age at which the children with HSP received MLS. This was the case in several previous studies on the possible predictors of outcome after MLS in children with CP. Švehlík, et al. found that children aged 10 to 12 years (and GMFCS level III) were the best responders to MLS at long-term FU [11]. In a different study by Švehlík, et al. it was stated that the older the child at the time of surgery, the better the long-term results [23]. In the current study, age of surgery showed no significant relation to post-operative GPS of the children with HSP at any follow-up moment. Likewise, GMFCS level also did also not relate to the long-term outcome of gait after MLS in a recent study by Dreher, et al. with a sample size of 231 children with CP and a follow-up period of 9-years [24].

In conclusion, we observed an overall improvement of gait in children with HSP at short- and middle-term, which was more pronounce for children who presented with flexion gait pattern in the hip and knee joint pre-operatively. We also noticed that some of the improvements that were observed at short-term, such as pelvic tilt ROM, were not maintained until medium-term FU and even significantly worsened later on. To preserve the early improvements, a substantial part of the patients required additional surgeries or Botulinum tox- 
Citation: Prinsen S, Buyck C, Beukelaer N, et al. (2020) The Effect of Multilevel Surgery on the Gait Pattern of Children with Hereditary Spastic Paraplegia, are there Differences in Outcome Compared to Those with Bilateral Spastic Cerebral Palsy?. J Orthop Surg Tech 3(2):179-190

in-A-injections. Age of surgery showed no crucial relation to the outcome of the children with HSP in our study and there are no essential differences found in outcome post-MLS between children with HSP and children with CP. Yet, but further research with larger sample sizes is required. We can cautiously concluded that MLS in children with HSP are not essentially different than the ones performed on children with CP. Naturally, every child needs to be treated individually, based on their individual 3DGA results, results of clinical examination and clinical interpretation.

\section{Conflicts of Interest}

The author has no conflicts of interest to report.

\section{References}

1. Blackstone Craig (2018) Hereditary spastic paraplegia. Handbook of clinical neurology 148: 633-652.

2. Dennis SC, NE Green (1988) Hereditary Spastic Paraplegia. Journal of pediatric orthopedics 8: 413-417.

3. Salinas Sara, Christos Proukakis, Andrew Crosby, et al. (2008) Hereditary spastic paraplegia: Clinical features and pathogenetic mechanisms. The Lancet Neurology 7: 1127-1238.

4. Peter Rosenbaum, Nigel Paneth, Alan Leviton, et al. (2007) A Report: The definition and classification of cerebral palsy April 2006. Developmental Medicine and Child Neurology Supplement 109: 8-14.

5. Michael C Fahey, Alastair H Maclennan, Doris Kretzschmar, et al. (2017) The genetic basis of cerebral palsy. Developmental Medicine and Child Neurology 59: 462-469.

6. Josef Finsterer, Wolfgang Löscher, Stefan Quasthoff, et al. (2012) Hereditary spastic paraplegias with autosomal dominant, recessive, X-linked, or maternal trait of inheritance. Journal of the Neurological Sciences 318: 1-18.

7. Kerr Graham H, P Selber (2003) Musculoskeletal aspects of cerebral palsy. The Journal of Bone and Joint Surgery British 85: 157-166.

8. Lamberts, Robert $P$, Marlette Burger, Jacques du Toit, et al. (2016) A systematic review of the effects of single-event multilevel surgery on gait parameters in children with spastic cerebral palsy. PLoS One 11: e0164686.

9. Jennifer L McGinley, Fiona Dobson, Rekha Ganeshalingam, et al. (2012) Single-event multilevel surgery for children with cerebral palsy: A systematic review. Developmental Medicine and Child Neurology 54: 117-128.

10. Luigi Piccinini, Veronica Cimolin, Maria Grazia D'Angelo, et al. (2011) 3D gait analysis in patients with hereditary spastic paraparesis and spastic diplegia: A kinematic, kinetic and EMG comparison. European Journal of Paediatric Neurology 15: 138-145.
11. Svehlik M, G Steinwender, T Lehmann, et al. (2016) Predictors of outcome after single-event multilevel surgery in children with cerebral palsy: A retrospective ten-year follow-up study. The Bone \& Joint Journal 98-B: 278-281.

12. Thomason Pam, Paulo Selber, H Kerr Graham (2013) Single event multilevel surgery in children with bilateral spastic cerebral palsy: A 5 year prospective cohort study. Gait \& Posture 37: 23-28.

13. Gough Martin, Linda C Eve, Richard O Robinson, et al. (2004) Short-term outcome of multilevel surgical intervention in spastic diplegic cerebral palsy compared with the natural history. Developmental Medicine and Child Neurology 46: 91-97.

14. Bohannon RW, MB Smith (1987) Interrater reliability of a modified ashworth scale of muscle spasticity. Physical Therapy 67: 206-207.

15. Haugh AB, AD Pandyan, GR Johnson (2006) A systematic review of the tardieu scale for the measurement of spasticity. Disability and Rehabilitation 28: 899-907.

16. Richard Baker, Jennifer L McGinley, Michael H Schwartz, et al. (2009) The gait profile score and movement analysis profile. Gait \& Posture 30: 265-569.

17. McMulkin Mark L, Bruce A MacWilliams (2015) Application of the gillette gait index, gait deviation index and gait profile score to multiple clinical pediatric populations. Gait \& Posture 41: 608-612.

18. Dequeker Griet, Anja Van Campenhout, Hilde Feys, et al. (2018) Evolution of self-care and functional mobility after single-event multilevel surgery in children and adolescents with spastic diplegic cerebral palsy. Developmental Medicine and Child Neurology 60: 505-512.

19. Campenhout, A van, P Neut, E Papageorgiou, et al. (2016) Natural evolution of gait in children with hereditary spastic paraplegia. Gait \& Posture 49: 189.

20. Saglam Yavuz, N Ekin Akalan, Yener Temelli, et al. (2016) Femoral derotation osteotomy with multi-level soft tissue procedures in children with cerebral palsy: Does it improve gait quality?. Journal of Childrens Orthopaedics 10: 41-48.

21. CY Chung, SH Lee, IH Choi, et al. (2008) Residual pelvic rotation after single-event multilevel surgery in spastic hemiplegia. The Journal of Bone and Joint Surgery British 90: 1234-1238.

22. Rutz Erich, Richard Baker, Oren Tirosh, et al. (2013) Are results after single-event multilevel surgery in cerebral palsy durable?. Clinical Orthopaedics and Related Research 471: 1028-1038.

23. Martin Svehlík, Gerhard Steinwender, Tanja Kraus, et al. (2011) The influence of age at single-event multilevel surgery on outcome in children with cerebral palsy who walk with flexed knee gait. Developmental Medicine and Child Neurology 53: 730-735.

24. Thomas Dreher, Pam Thomason, Martin Švehlík, et al. (2018) Long-term development of gait after multilevel surgery in children with cerebral palsy: A multicentre cohort study. Developmental Medicine and Child Neurology 60: 88-93.

DOI: $10.36959 / 453 / 544$

Copyright: (C) 2020 Prinsen S, et al. This is an open-access article distributed under the terms of the Creative Commons Attribution License, which permits unrestricted use, distribution, and reproduction in any medium, provided the original author and source are credited. 